\title{
Cross - shareholding Analysis of China 's Listed Companies by Sector based on Social Network Perspective
}

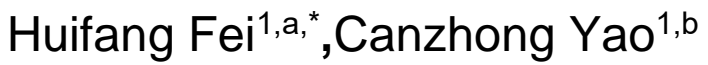 \\ ${ }^{1}$ school of economics and commerce, South China University of Technology, guangzhou, \\ guangdong, china \\ aashleyfeifang@163.com, b13676952979@163.com
}

keywords: Cross-shareholdings, social network, QAP analysis, Performance Impact Analysis.

\begin{abstract}
Taking the cross shareholdings(CSH) A-share firms from Shanghai and Shenzhen stock markets from 2006 to 2014 as research samples, this study constructs corporate network and industrial network of CSH from the perspective of social network. QAP analysis is used to explore the influence factors of industry shareholding relationship, and Multi-regression analysis is used to explore the influence of network location index on different industry performance. The results show that CSH of industrial relationship tends to occur among industries having weak correlation, similar industrial profitability and operating capacity. CSH relationship is irrelevant to whether these enterprises are in the same industries or not. The network location index only has significant influence on the performance of the public utility industry and the information technology industry with degree centrality and betweenness centrality having a significant positive effect for the former and betweenness centrality having a significant negative impact for the latter.
\end{abstract}

\section{基于社会网络视角的分行业中国上市公司交叉持股分析}

\author{
费慧芳 $1, a$ 姚灿中 $1, b$ \\ 1 华南理工大学经济与贸易学院,广州, 广东, 中国 \\ aashleyfeifang@163.com, b13676952979@163.com
}

关键词：交叉持股；社会网络；QAP 分析；绩效影响分析

中文摘要：本文基于社会网络视角, 以 2006-2014 年沪深 A 股涉及交叉持股的上市公司为样 本, 分别构建公司和行业的交叉持股网络。使用 QAP 的分析方法探究行业持股关系建立的影 响因素，使用多元回归分析网络位置对不同行业绩效的影响。结果表明，行业交叉持股倾向 于发生在行业相关性较弱, 以及盈利能力和营运能力相似的行业之间; 网络位置的两个衡量 指标度中心性和介数中心性对公用事业行业绩效有显著的正向促进作用; 介数中心性对信息 技术行业则有显著的负影响力。

\section{1. 引言}

交叉持股是指两个或两个以上的公司相互持有对方的股份而形成的公司间相互投资的现 象 ${ }^{[1-2]}$ 。国际上对于交叉持股的研究起步较早, 主要从公司治理角度分别对交叉持股的动因和 效应两个方面进行研究。由于中国公司间交叉持股的现象出现较晚, 因此国内对于交叉持股 的研究, 主要集中于法律规制、“双刃剑” 效应、财务会计政策、现状与特征等方面的研究, 对动因与效应的研究相对较少, 且多数以定性分析为主, 量化研究比较少见。 
复杂网络的分析方法 ${ }^{[3-4]}$ 为交叉持股的分析提供了更新的视角。复杂网络作为一门新兴发 展的学科, 它的主要研究方法是把不同的对象看作网络中的节点,对象之间的相互联系为节点 间的边,在此基础上来研究网络的拓扑特性。本文则立足于该视角, 以参与交叉持股的公司为 节点，构建公司交叉持股网络，并首次对所有节点基于行业进行分类，构建行业交叉持股网 络, 在此基础上进行量化分析。文章采用 QAP 的研究方法来测度不同行业间建立持股关系的 内在影响因素，并基于多元回归的方法测度网络位置指标对不同行业经营绩效的影响。

\section{2. 数据的选取与网络的构建}

\section{1 数据的选取}

本文对 2006 年至 2014 年间全部沪深 A 股上市公司之间的交叉持股行为进行研究，交叉 持股数据及参与企业的基本面数据来源于 WIND 数据库。本文利用数据库提供的数据计算出 相关指标,作为研究样本公司所需的数据。

\section{2 交叉持股网络的构建方法}

网络构建方法是，如果公司 $\mathrm{A}$ 持有公司 $\mathrm{B}$ 的股份，则划一条从 A 出发指向 $\mathrm{B}$ 的有向线 段, 一个公司每多持有一个公司的股份, 我们称之为出度 $\left(K_{\text {out }}\right)$ 增加 1 , 被其他公司持有一 次, 入度 $\left(K_{\text {in }}\right)$ 增加 1, 所有参与公司之间错综复杂的持股关系用这种方式反映出来, 表现为 一个网络图，图 1（左）为 2014 年的公司交叉持股网络图，不同的颜色代表公司所处的不 同行业（基于万德三级行业分类），节点越大代表了与之建立持股关系的节点数越多。把图 1 中相同颜色代表同一行业的节点融合在一起, 聚集成 62 个节点, 代表 62 个行业。两个行 业节点之间的连边的数值代表二者发生的所有持股关系总和, 连边越粗则代表两个行业的公 司间持股关系数越多。这样就构成了图 1（右）的行业交叉持股网络图。

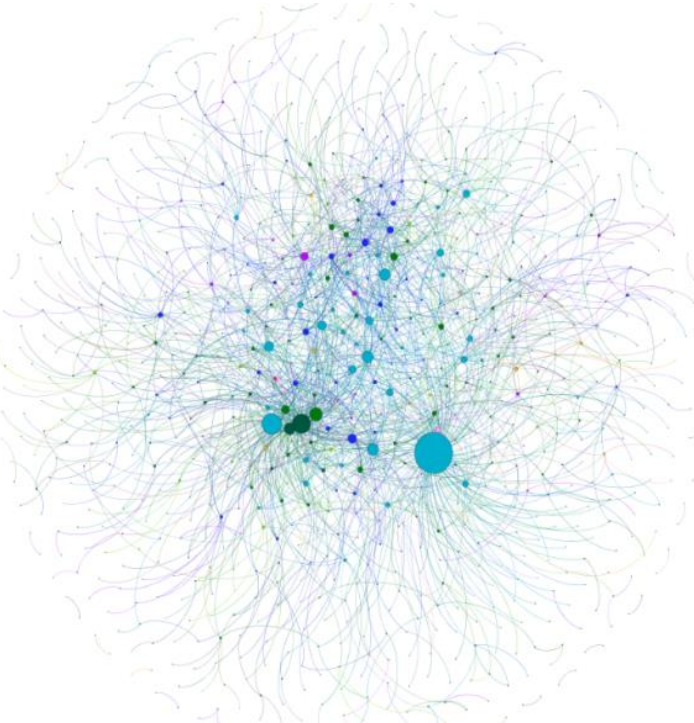

2014 年公司交叉持股网络

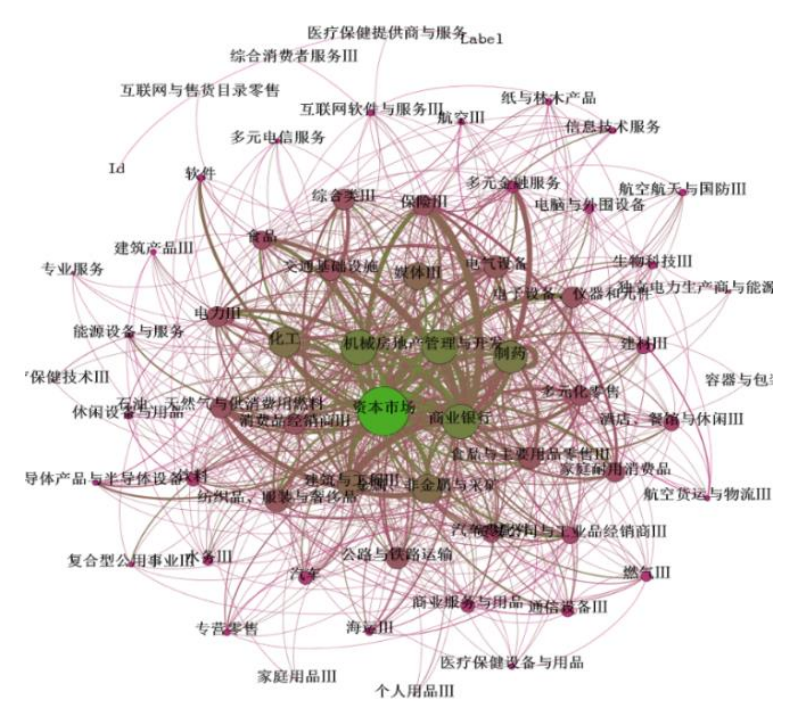

2014 年行业交叉持股网咯

图 1 公司交叉持股网络拓扑结构

\section{3. 行业交叉持股的QAP回归分析}

公司之间的交叉持股行为大部分发生在不同行业之间，行业内部的交叉持股行为较少。 表 1 为 2006-2014 年交叉持股行业内公司的连接占比。根据表 1 可计算得到, 行业内交叉持 股行为平均占比为 7\%, 说明公司之间的交叉持股约 $90 \%$ 都发生于行业间, 行业内部建立持 股关系的比例实际较低。 
表 1 行业内连接占比

\begin{tabular}{cccccccccc}
\hline 年份 & 2006 & 2007 & 2008 & 2009 & 2010 & 2011 & 2012 & 2013 & 2014 \\
\hline 行业内连接占比 & $7.61 \%$ & $6.20 \%$ & $6.14 \%$ & $6.14 \%$ & $6.42 \%$ & $6.21 \%$ & $7.76 \%$ & $8.00 \%$ & $8.43 \%$ \\
\hline
\end{tabular}

为了揭示行业间发生交叉持股行为的影响因素, 我们采用 $\mathrm{QAP}^{[5]}$ 方法, 对交叉持股矩阵 与行业相关性矩阵进行回归分析。

\subsection{QAP 方法}

QAP 以重新抽样为基础, 是一种随机化检验的方法, 其研究的对象都是 “关系” 数据。 通过对方阵中各个值进行比较, 计算两个矩阵之间的相关系数, 同时以对矩阵数据的置换为 基础对系数进行非参数检验。以同一数据为基础的 QAP 回归分析所得的 $R^{2}$ 系数相较 OLS 估计通常要低一些[6]，马丽莎, 王建琼, 董大勇和钟勇 ${ }^{[7]}$ 运用 QAP 方法, 分析我国上市公司的 交叉持股矩阵和股价相关系数矩阵的关系时, $R^{2}$ 系数为 0.023 。在进行回归分析估计时, 首 先针对自变量矩阵和因变量矩阵对应的长向量元素进行常规的多元回归分析; 然后对因变量 矩阵的各行与各列同时进行随机置换并重新计算回归，保存所有的系数值以及判定系数值; 最后在一定抽样次数限制下重复进行随机置换以便估计统计量的标准误。本文采用 QAP 方 法对矩阵和矩阵进行估计，在回归分析时将抽样次数设置为 200 次。

\section{2 指标选取}

盈利能力指标主要包括营业利润率、成本费用利润率、盈余现金保障倍数、总资产收益 率、净资产收益率和资本收益率六项，本文选取总资产收益率来衡量行业的盈利能力。

总资产收益率=净利润 $\div$ 平均资产总额 $\times 100 \%$

总资产收益率指标集中体现了资产运用效率和资金利用效果之间的关系。在资产总额一 定的情况下, 利用总资产收益率指标可以分析不同行业盈利的稳定性和持久性, 确定所面临 的风险。

营运能力的衡量来自于流动资产周转情况、固定资产周转情况和总资产周转情况。考虑 到行业性质决定的固定资本投入的外生性差异, 流动资产周转情况是揭示行业资产质量具有 代表性的因素。流动性资产周转情况的衡量指标包括存货周转率和应收账款周转率, 因为并 非所有行业都有存货，故选取应收账款周转率来衡量行业的营运能力。

应收账款周转率=主营业务收入净额 $\div$ 应收账款平均余额

应收账款在流动资产中具有举足轻重的地位。应收账款周转率越高,表明收账速度快, 平均收账期短, 坏账损失少, 资产流动快, 营运能力强。但从另一方面说, 如果应收账款周 转天数太短, 则表明该类行业奉行较紧的信用政策, 付款条件过于苛刻, 这样会限制销售量 的扩大，特别是当这种限制的代价（机会收益）大于㻌销成本时，会影响到整体盈利水平。 故该指标可以一定程度上衡量不同行业的营运特征差异。

偿债能力分析反映上市公司偿债能力的指标, 主要有流动比率、速动比率、现金比率、 资本周转率、清算价值比率和利息支付倍数等, 其中利息支付倍数综合反映了行业偿债能力 的保障程度, 即负债经营的财务风险程度。本文选取已获利息倍数来衡量行业的偿债能力。

已获利息倍数=(净利润 + 利息费用 + 所得税费用 $) \div$ 利息费用。

一般情况下, 已获利息倍数越高, 长期偿债能力越强, 反之则偿债能力较弱。故该指标 可以在一定程度上衡量不同行业经营的财务风险程度。

行业与行业之间的相关性指标采用 2013-2014 年这一时间段内收益率相关系数的 $62 \times 62$ 矩阵 Cor 来表示，每一个矩阵元素代表两两之间的相关系数。其元素取值为:

$$
\operatorname{cor}_{i, j}=\frac{\sum\left(r_{i, t}-\bar{r}_{i}\right)\left(r_{j, t}-\bar{r}_{j}\right)}{\sqrt{\sum\left(r_{i, t}-\bar{r}_{i}\right)^{2}} \sum\left(r_{j, t}-\bar{r}_{j}\right)^{2}} i, j=1,2,3 \ldots \ldots \ldots 62
$$


其中, $r_{i, t} 、 r_{j, t}$ 为行业 $\mathrm{i}, \mathrm{j}$ 在 $\mathrm{t}$ 日之内的对数收益率。

相应指标与矩阵的构造方法为:

表 2 分析指标体系与矩阵构建

\begin{tabular}{|c|c|c|}
\hline & 指标 & 计算方法 \\
\hline 行业相关性 & $\begin{array}{l}\text { 行业相关性矩 } \\
\text { 阵 }\end{array}$ & 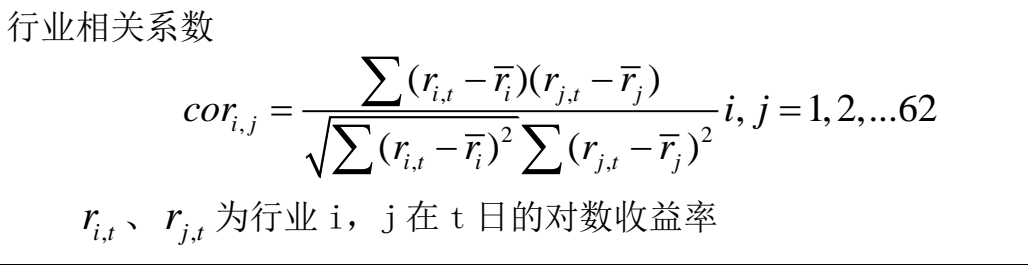 \\
\hline 盈利能力相似性 & $\begin{array}{l}\text { 总资产收益率 } \\
\text { 差异矩阵 }\end{array}$ & 盈利能力差异值 $=\left|\ln \left(\frac{\text { 行业 } \mathrm{i} \text { 的总资产收益率 }}{\text { 行业 } \mathrm{j} \text { 的总资产收益率 }}\right)\right| i, j=1,2, \ldots 62$ \\
\hline 营运能力相似性 & $\begin{array}{l}\text { 应收账款周转 } \\
\text { 率差异矩阵 }\end{array}$ & 营运能力差异值 $=\left|\ln \left(\frac{\text { 行业 } \mathrm{i} \text { 的应收账款周转率 }}{\text { 行 } \mathrm{j} \text { 的应收账款周转率 }}\right)\right| i, j=1,2, \ldots 62$ \\
\hline 偿债能力相似性 & $\begin{array}{l}\text { 已获利息倍数 } \\
\text { 差异矩阵 }\end{array}$ & 偿债能力差异值 $=\left|\ln \left(\frac{\text { 行业 } \mathrm{i} \text { 的已获利息倍数 }}{\text { 行业 } \mathrm{j} \text { 的已获利息倍数 }}\right)\right| i, j=1,2, \ldots 62$ \\
\hline
\end{tabular}

其中财务指标差异值越小，表明行业 $\mathrm{i} 、 \mathrm{j}$ 之间的某一财务指标越相近。

\section{3 结果解释}

QAP 分析结果如下:

表 3 行业交叉持股关系矩阵与其他四个关系矩阵的相关性

\begin{tabular}{ccc}
\hline & 相关系数 & significa \\
\hline 行业相关矩阵 & -0.2750 & 0.116 \\
总资产收益率差异矩阵 & 0.1520 & $0.0330 * *$ \\
已获利息倍数差异矩阵 & 0.0380 & 0.3140 \\
应收账款周转率差异矩阵 & -0.1450 & $0.0230 * *$ \\
\hline
\end{tabular}

由表 3 分析结果可知, 代表行业相关性与偿债能力相似性的指标没有通过显著性检验, 代表盈利能力相似性和营运能力相似性的指标在 0.05 的显著性水平下显著。行业相关性未 通过显著性检验一定程度上验证了我们之前的推测, 即交叉持股关系的发生与二者是否为同 一行业并无关联。而盈利能力相似性和营运能力相似性在多大程度上影响了行业之间的交叉 持股的选择呢, 在此基础上我们进行 QAP 回归分析, 结果如下:

表 4 QAP 回归分析得到的各变量回归系数及检测指标

\begin{tabular}{cccc}
\hline 变量 & 非标准化回归系数 & 标准化回归系数 & 显著性概率值 \\
截距 & 112.591 & 0.000 & - \\
总资产收益率差异矩阵 & 1.284 & 0.098 & 0.045 \\
应收账款周转率差异矩阵 & -1.287 & -0.071 & 0.072 \\
\hline
\end{tabular}

该回归调整后的判定系数 $R^{2}$ 为 0.105 , 说明两个矩阵变量可以解释交叉持股关系建立因 素的 $10.5 \%$ 。总资产收益率差异矩阵在 $5 \%$ 水平上显著, 说明盈利能力相似的行业之间容易 建立交叉持股关系。应收账款周转率相似性在 $10 \%$ 水平上显著, 说明说明营运能力相似的行 业之间容易建立交叉持股关系。行业与行业之间的持股关系倾向于发生在行业相关性较弱以 及盈利能力和营运能力相似的行业之间。 


\section{4. 网络位置指标对不同行业绩效的影响分析}

企业的一切决策都是为了提高企业的绩效水平，从而提升企业的竞争力，企业交叉持股 网络的建立也是出于同样的目的。而这种关系的建立不一定对所有的企业都是利好的, 我们 可以从公司交叉持股网络中获取有用的信息, 来帮助企业做决策时多一个参考因素。前文我 们分析了不同行业之间建立交叉持股关联的影响因素，那么交叉持股对不同行业的绩效影响 如何呢?

\section{1 企业经营绩效}

企业绩效为被解释变量, 用总资产净利率（ROA）进行度量, 总资产净利率等于净利润 与平均资产总额的比值, 净利润=营业利润+营业外收入一营业外支出一税收

\section{2 网络位置指标}

(1)度中心性（Degree centrality）

如果一个公司与其他很多公司有直接的交叉持股关系，该公司就居于中心地位，从而拥 有较大的影响力。居于中心地位的公司往往与其它公司有多种关联, 居于边缘地位的公司并 非如此, 基于该种思路来测量一个点的度数中心度, 可以仅根据与该点有直接关系的点的数 目:

$$
C_{R D}(X)=\frac{\left(K_{\text {in }}+K_{\text {out }}\right)}{2 n-2}
$$

其中, $\mathrm{n}$ 是网络的规模, $K_{\text {in }}$ 表示 $\mathrm{X}$ 的入度, $K_{\text {out }}$ 表示 $\mathrm{X}$ 的出度。

(2)、介数中心性（Betweenness centrality）

有的公司，虽然连接公司较少，但是处于许多公司相连接的交往网络路径上，可以认为 此公司居于重要地位, 因为它具有影响其它公司之间交往的能力。在这种思路的指导下测量 一个点的介数中心性, 它测量的是交叉持股网络中某个公司控制其他公司联系路径的程度:

$$
C_{A B i}=\sum_{j}^{n} \sum_{k}^{n} g_{j k}(i) / g_{j k} j \neq k \neq i \text { 并且 } j<k
$$
的概率。

其中, $\mathrm{j}$ 和 $\mathrm{k}$ 之间存在的捷径数目用 $g_{j k}$ 来表示, $g_{j k}(i) / g_{j k}$ 表示 $\mathrm{i}$ 处于 $\mathrm{j}$ 和 $\mathrm{k}$ 之间捷径上

\section{3 控制变量}

除了解释变量之外, 影响企业绩效的因素还有很多, 本文选取了公司规模、资产负债 率、股权集中度、净资产增长率，具体的所有变量说明见表

\begin{tabular}{|c|c|c|}
\hline 类型 & 名称及简称 & 定义及说明 \\
\hline 被解释变量 & 总资产净利率（ROA） & $\begin{array}{l}\text { ROA=净利润/总资产; 净利润 }=\text { 营业利润+营业外收入－营业外支出 } \\
\text { 一税收 }\end{array}$ \\
\hline 解释变量 & $\begin{array}{l}\text { 度数中心性 }(\mathrm{DC}) \\
\text { 介数中心性 }(\mathrm{BC})\end{array}$ & $\begin{array}{l}\text { 反应与整体网络成员联系的紧密程度 } \\
\text { 反应控制其他公司联系路径的程度, 既其所处在网络中的要塞程度 }\end{array}$ \\
\hline 控制变量 & $\begin{array}{l}\text { 公司规模 (SIZE) } \\
\text { 资产负债情况 (LEV) } \\
\text { 净资产增长率 (NAV) } \\
\text { 股权集中度 (EOS) }\end{array}$ & 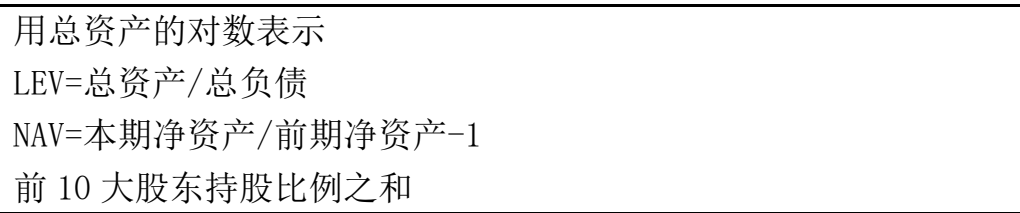 \\
\hline
\end{tabular}

表 5 回归变量 


\section{4.样本分布与回归模型}

多元回归方程如下:

$$
\begin{aligned}
& \mathrm{ROA}=\beta_{0}^{1}+\beta_{1}^{1} \mathrm{SIZE}+\beta_{2}^{1} L E V+\beta_{3}^{1} \mathrm{EOS}+\beta_{1} \mathrm{DC}+\varepsilon_{1} \\
& \mathrm{ROA}=\beta_{0}^{2}+\beta_{1}^{2} \mathrm{SIZE}+\beta_{2}^{2} L E V+\beta_{3}^{2} \mathrm{EOS}+\beta_{2} \mathrm{BC}+\varepsilon_{2}
\end{aligned}
$$

其中, 公式 (4) 和 (5) 分别是度中心性（DC）和介数中心性（BC）作为解释变量建 立的多元回归模型, $\beta_{1}$ 和 $\beta_{2}$ 为各自的影响系数。

对节点基于 Wind 一级行业分类标准进行划分，具体的样本分布情况如下表所示：

表 6 交叉持股行业的样本分布

\begin{tabular}{lcccccccccc}
\hline 行业 & 2006 & 2007 & 2008 & 2009 & 2010 & 2011 & 2012 & 2013 & 2014 & 样本总数 \\
\hline 材料 & 112 & 147 & 157 & 142 & 163 & 162 & 147 & 145 & 154 & 1329 \\
电信服务 & 2 & 1 & 3 & 2 & 2 & 2 & 1 & 1 & 3 & 17 \\
工业 & 167 & 188 & 180 & 207 & 263 & 249 & 240 & 239 & 245 & 1978 \\
公用事业 & 45 & 46 & 44 & 46 & 48 & 49 & 47 & 54 & 58 & 437 \\
金融 & 110 & 120 & 123 & 135 & 128 & 118 & 119 & 122 & 121 & 1096 \\
可选消费 & 143 & 150 & 159 & 155 & 170 & 160 & 178 & 175 & 187 & 1477 \\
能源 & 23 & 30 & 30 & 28 & 35 & 34 & 34 & 35 & 36 & 285 \\
日常消费 & 51 & 59 & 61 & 59 & 71 & 70 & 69 & 70 & 74 & 584 \\
信息技术 & 62 & 78 & 84 & 98 & 105 & 102 & 95 & 104 & 113 & 841 \\
医疗保健 & 49 & 57 & 56 & 64 & 66 & 72 & 74 & 87 & 72 & 597 \\
\hline
\end{tabular}

各个行业样本量分别是：材料 1303 个、电信服务业 17 个、工业 1962 个、公用事业 437 个、金融业 1094 个、可选消费业 1459 个、能源业 277 个、日常消费业 576 个、信息技术业 820 个、医疗保健业 588 个。电信服务业由于样本量太小, 不具有研究条件, 故不对该行业进 行研究。度中心性指标和介数中心性指标与净资产收益率之前的相关系数较低, 分别为 0.022 和 0.018, 说明它们之间的线性关系可能不显著。多元回归结果如下:

\begin{tabular}{|c|c|c|c|c|c|c|c|c|}
\hline 行业 & & $\beta_{0}^{1}$ & SIZE & LEV & EOS & NAV & $\mathrm{DC}$ & $R^{2}$ \\
\hline \multirow{2}{*}{ 材料 } & 系数 & 0.117 & -0.007 & -0.053 & 0.045 & 0.101 & -0.001 & \multirow{2}{*}{0.187} \\
\hline & sig & $.000 * * *$ & $.035 * *$ & $.000 * * *$ & $.000 * * *$ & $.000 * * *$ & 0.899 & \\
\hline \multirow{2}{*}{ 工业 } & 系数 & 0.431 & -0.041 & -0.002 & 0.033 & 0.09 & 0.011 & \multirow{2}{*}{0.166} \\
\hline & sig & $.000 * * *$ & $.000 * * *$ & 0.269 & 0.33 & $.011 * *$ & 0.295 & \\
\hline \multirow{2}{*}{ 公用事业 } & 系数 & 0.09 & -0.011 & 0.028 & 0.051 & 0.009 & 0.022 & \multirow{2}{*}{0.256} \\
\hline & sig & $008 * * *$ & $.004 * * *$ & $.000 * * *$ & $.000 * * *$ & $.017 * *$ & $.001 * * *$ & \\
\hline \multirow{2}{*}{ 金融 } & 系数 & 1.952 & -0.189 & 0.004 & 0.054 & 0.002 & 0.045 & \multirow{2}{*}{0.117} \\
\hline & sig & $001 * * *$ & $.002 * * *$ & $.032 * *$ & 0.857 & 0.616 & 0.363 & \\
\hline \multirow{2}{*}{ 可选消费 } & 系数 & 1.374 & -0.137 & -0.004 & -0.004 & 0.033 & -0.002 & \multirow{2}{*}{0.115} \\
\hline & sig & $.000 * * *$ & $.000 * * *$ & 0.499 & 0.97 & $.091 *$ & 0.95 & \\
\hline \multirow{2}{*}{ 能源 } & 系数 & 0.04 & 0.003 & -0.069 & 0.036 & 0.004 & 0.003 & \multirow{2}{*}{0.095} \\
\hline & sig & 0.452 & 0.627 & $.000 * * *$ & 0.115 & 0.215 & 0.625 & \\
\hline \multirow{2}{*}{ 日常消费 } & 系数 & -0.166 & 0.022 & -0.063 & 0.09 & 0.011 & 0.003 & \multirow{2}{*}{0.213} \\
\hline & sig & $000 * * *$ & $.000 * * *$ & $.000 * * *$ & $.000 * * *$ & $.000 * * *$ & 0.573 & \\
\hline \multirow{2}{*}{ 信息技术 } & 系数 & 0.524 & -0.053 & -0.006 & 0.065 & 0.006 & -0.015 & \multirow{2}{*}{0.201} \\
\hline & sig & $.000 * * *$ & $.000 * * *$ & 0.124 & $.000 * * *$ & $.001 * * *$ & 0.131 & \\
\hline \multirow{2}{*}{ 医疗保健 } & 系数 & -0.045 & 0.015 & -0.102 & 0.035 & 0.006 & 0.002 & \multirow{2}{*}{0.168} \\
\hline & sig & 0.188 & $.000 * * *$ & $.000 * * *$ & $.032 * * *$ & $.008 * * *$ & 0.75 & \\
\hline
\end{tabular}

表 7 网络位置对企业绩效的多元回归分析（1） 
表 8 网络位置对企业绩效的多元回归分析（2）

\begin{tabular}{|c|c|c|c|c|c|c|c|c|}
\hline 行业 & & $\beta_{0}^{2}$ & SIZE & LEV & E0S & NAV & $\mathrm{BC}$ & $R^{2}$ \\
\hline \multirow{2}{*}{ 材料 } & 系数 & 0.12 & -0.007 & -0.053 & 0.045 & 0.102 & 0.003 & \multirow{2}{*}{0.187} \\
\hline & sig & $.000 * * *$ & $.026 * *$ & $.000 * * *$ & $.000 * * *$ & $000 * * *$ & 0.524 & \\
\hline \multirow{2}{*}{ 工业 } & 系数 & 0.434 & -0.041 & -0.002 & 0.032 & 0.09 & 0.008 & \multirow{2}{*}{0.116} \\
\hline & sig & $.000 * * *$ & $.000 * * *$ & 0.267 & 0.338 & $.010 * * *$ & 0.347 & \\
\hline \multirow{2}{*}{ 公用事业 } & 系数 & 0.089 & -0.01 & 0.028 & 0.05 & 0.009 & 0.015 & \multirow{2}{*}{0.254} \\
\hline & sig & $.008 * * *$ & $.006 * * *$ & $.000 * * *$ & $.000 * * *$ & $.018 * *$ & $.002 * * *$ & \\
\hline \multirow{2}{*}{ 金融 } & 系数 & 1.918 & -0.184 & 0.004 & 0.052 & 0.002 & 0.02 & \multirow{2}{*}{0.116} \\
\hline & sig & $.001 * * *$ & $.002 * * *$ & $.032 * * *$ & 0.863 & 0.619 & 0.399 & \\
\hline \multirow{2}{*}{ 可选消费 } & 系数 & 1. 389 & -0.14 & -0.004 & 0.008 & 0.034 & 0.029 & \multirow{2}{*}{0.116} \\
\hline & sig & $.000 * * *$ & $.000 * * *$ & 0.5 & 0.941 & $.084 *$ & 0.326 & \\
\hline \multirow{2}{*}{ 能源 } & 系数 & 0.039 & 0.003 & -0.07 & 0.036 & 0.004 & 0.002 & \multirow{2}{*}{0.095} \\
\hline & sig & 0.446 & 0.588 & $.000 * * *$ & 0.115 & 0.205 & 0.59 & \\
\hline \multirow{2}{*}{ 日常消费 } & 系数 & -0.171 & 0.022 & -0.064 & 0.087 & 0.011 & -0.002 & \multirow{2}{*}{0.213} \\
\hline & sig & $.000 * * *$ & $.000 * * *$ & $.000 * * *$ & $.000 * * *$ & $000 * * *$ & 0.614 & \\
\hline \multirow{2}{*}{ 信息技术 } & 系数 & 0.537 & -0.054 & -0.006 & 0.062 & 0.006 & -0.026 & \multirow{2}{*}{0.206} \\
\hline & sig & $.000 * * *$ & $.000 * * *$ & 0.124 & $.000 * * *$ & $.002 * * *$ & $.007 * * *$ & \\
\hline \multirow{2}{*}{ 医疗保健 } & 系数 & -.044 & .015 & -.102 & .035 & .006 & .002 & \multirow{2}{*}{0.168} \\
\hline & sig & .197 & $.000 * * *$ & $.000 * * *$ & $.031 * *$ & $.007 * * *$ & .596 & \\
\hline
\end{tabular}

研究结果表明, 公用事业行业无论从度中心性角度还是从介数中心性角度来考量, 均在 0.01 的显著性水平下显著，影响力都为正。表明公用事业企业积极的去参与交叉持股，增大 度数中心性，以及在交叉持股网络中找到核心桥梁位置建立持股关系，增大介数中心性，均 可以有效地提高企业的绩效水平；此外，信息技术行业的介数中心性在 0.01 的显著性水平下 显著，且影响力为负。表明信息技术行业在构建交叉持股关系时，可以建立在对整个网络的 分析基础之上，避免与某些企业建立持股关系之后，使得自身处于许多公司相连接的交往网 络路径上, 从而具有较大介数中心性, 影响盈利水平。除此之外, 该两个指标对其他行业的 盈利水平均无显著性影响。

\section{5、结论:}

本文基于社会网络视角, 以 2006-2014年沪深 A 股所有涉及交叉持股的上市公司为样本, 分别构建公司交叉持股网络和行业交叉持股网络。使用 QAP 的分析方法探究行业持股关系建 立的影响因素，使用多元回归的方法探究交叉持股对不同行业的绩效影响。结果表明,行业交 叉持股倾向于发生在行业相关性较弱, 以及盈利能力和营运能力相似的行业之间, 交叉持股 行为与是否同一行业并无显著性关联。多元回归的结果显示，网络位置指标对除了公共事业 和信息技术行业之外的其他行业影响并不显著, 公用事业行业无论从度中心性角度还是从介 数中心性角度来考量, 均有显著的正向促进作用; 介数中心性对信息技术行业的企业经营绩 效则有显著的负影响力。

据此，我们提出以下建议，在上市公司数据公开透明的前提下，公共事业和信息技术行 业在准备参与交叉持股时，可以据此构建交叉持股网络，参考网络指标来辅助自己做出持股 或者被持股的决策，从而降低自己的投资风险，提高经营绩效。公共事业单位可以积极的参 与交叉持股, 且选择网络中的要塞位置会更有利于公司绩效的提高, 而信息技术行业则要避 免在网络中处于要塞位置，规避因参与交叉持股而影响公司的运作效率。 


\section{References}

[1] Osano, H.. Intercorporate shareholdings and corporate control in the Japanese firm [J]. Journal of Banking \& Finance, 1996, 20(6):1047-1068.

[2] Berglof and Perotti, E.. The Governance Structure of the Japanese Financial Keiretsu [J]. Journal of Law and Economics, 1994, 36(2):259-284.

[3] Barabasi A L. and Albert R.. Emergence of scaling in random networks[J]. Science, 1999, 286(5439):509-512.

[4] Newman, M. E.. Clustering and preferential attachment in growing networks[J]. Physical Review E, 2001, 64(2): 025102 .

[5] L.Jun. Introduction to social network analysis [M]. Beijing: Social Science Literature Press, 2007,73-86.

[6] X. Qun-Ying and L. Hui-Jun. The theoretical re examination of inter provincial labor migration based on QAP algorithm [J]. Chinese Population Science, 2007,04:26-33+95.

[7] M. Li-Sha and W. Jian-Qiong. Is cross shareholdings affect stock price linkage? [J]. Finance and Trade Economics, 2014,04:59-68. 